\title{
Examination of the Effectiveness of Far-field Mathematical Absorber Reflection Suppression in a CATR Through Electromagnetic Simulation
}

\author{
S.F. Gregson ${ }^{1,2^{*}}$, C.G. Parini ${ }^{2}$, A.C. Newell ${ }^{1}$ \\ ${ }^{1}$ NSI-MI Technologies, 19730 Magellan Drive, Torrance, California, USA \\ ${ }^{2}$ Queen Mary University of London, London, UK \\ *stuart.gregson@qmul.ac.uk
}

\begin{abstract}
For more than a decade now, a measurement and post-processing technique involving modal filtering, named Mathematical Absorber Reflection Suppression (MARS), has been used very successfully to identify and subsequently extract range reflections in spherical, cylindrical and planar near-field antenna test systems and far-field and compact antenna test ranges. Much of the early work concentrated on verification through experimental testing however some additional validation was performed using computational electromagnetic simulations. These considered first far-field and subsequently near-field cases. The recent development of an accurate, flexible electromagnetic simulation tool that enables the simulation of "measured" far-field pattern data as obtained from using a compact antenna test range (CATR) has, for the first time, permitted the careful verification of the far-field MARS technique for a specified AUT and CATR combination. This paper presents simulated "measured" far-field antenna pattern data in the presence of a large scatterer and then verifies the successful extraction of the scattering artefacts. In addition to considering range reflections, feed spill-over is also treated. Results are presented and discussed.
\end{abstract}

\section{Introduction}

The popularity of collimating antenna measurements (the so-called Compact Antenna Test Range - CATR) stems from a number of factors. These generally include: the simplicity with which far-field parameters can be obtained from the experimental equipment, the absence of the need to undertake intensive mathematical and numerical analysis, the ability to acquire boresight and pattern cut-data efficiently without the need to first acquire complete twodimensional pattern data, the ability to perform real-time measurements using arbitrary waveforms, and often the greatest attraction being the ability to relocate testing indoors to a highly repeatable, electromagnetically quiet, anechoic test environment.

However, no test environment is perfectly adiabatic and it is generally found that reflections within the test site can be one of the largest sources of measurement error within the uncertainty budget [2] with direct collimating ranges being perhaps the most susceptible to these sorts of contaminants [1, 3, 4]. The recent completion of an extensive validation campaign for a new CATR electromagnetic simulation tool that permits the calculation of the error to signal level for a specified AUT and CATR combination [5] provides the possibility of further detailed verification of the far-field FF-MARS technique. Thus, in addition to being able to provide the conventional CATR QZ performance metrics such as amplitude taper, amplitude peak-to-peak ripple and phase peak-to-peak ripple [1], it is also possible to provide full-sphere simulated far-field "measured" data for a given CATR AUT combination including known measurement errors. The following sections describe the CATR system being modelled [6], present simulated CATR quiet-zone (QZ) performance parameters before presenting the results of a simulated CATR measurement of a pyramidal horn in the presence of a strong spurious scattered signal. These simulated "measured" patterns are then compensated using standard FF-MARS post-processing [7].

\section{Overview of the measurement simulation}

To illustrate the simulation and measurement concept, we take the example of a sector-shaped single offset reflector CATR that has no edge treatment. This system can be seen presented schematically Figure $1[1,6]$. This $3 \mathrm{~m}$, 18 panel CATR forms the $8 \mathrm{GHz}$ to $60 \mathrm{GHz}$ CATR facility in Queen Mary University of London's (QMUL) Antenna Measurement Laboratory. The offset parabolic reflector has an overall surface accuracy of approximately 80 microns and a $5.4 \mathrm{~m}$ focal length.

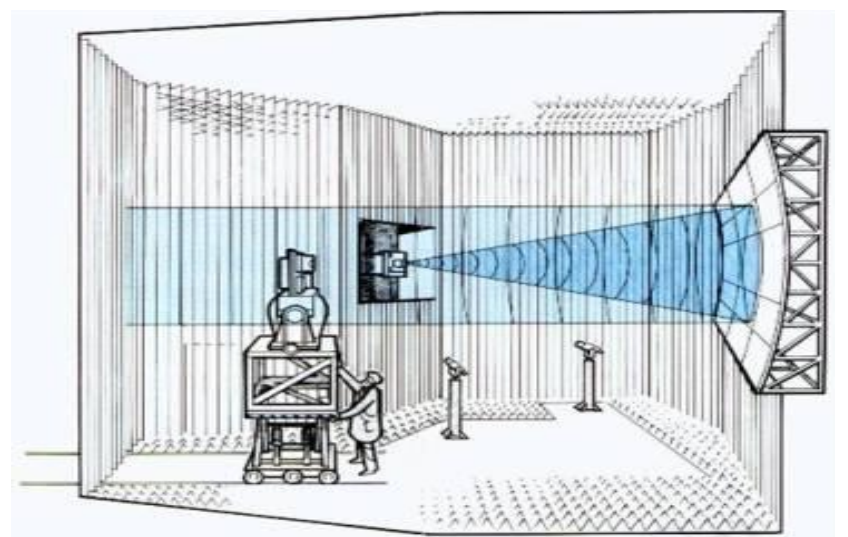

Fig. 1. Schematic representation Queen Mary University of London's sector-shaped single offset reflector CATR.

In our EM model the surface profile of the point source CATR is assumed to be comprised of an ideal concave paraboloidal surface, with the reflector edge having no treatment and being modelled as a perfect metallic knifeedge. Surface imperfections and edge castellation can be included within the analysis; however, for the present study 
these are thought to constitute second order effects and so were not incorporated although much work has been done previously on this topic [1]. The phase centre of the range's corrugated horn feed is situated at the focus of the offset parabolic reflector with the feed being tilted to an angle of $28^{\circ}$ in azimuth so that the edges of the reflector are seen at circa $\pm 14^{\circ}$ about this direction. At $8 \mathrm{GHz}$ the corrugated feed has an aperture size of $3.39 \lambda$ and presents an edge illumination in the azimuth plane of approximately $-5.0 \mathrm{~dB}$. This particular configuration was selected as it modelled the CATR that was initially used to experimentally verify the FF-MARS technique $[1,4]$ thereby admitting the possibility of further verification between simulation and measurement. The simulated amplitude and phase of the field illuminating the reflector is presented below in Figure 2 and 3 respectively. The cross shown in Figure 2 represents the maximum field intensity and can be seen to be located in the centre of the reflector. In Figure 3, the spherical phase factor has been suppressed for the sake of clarity.

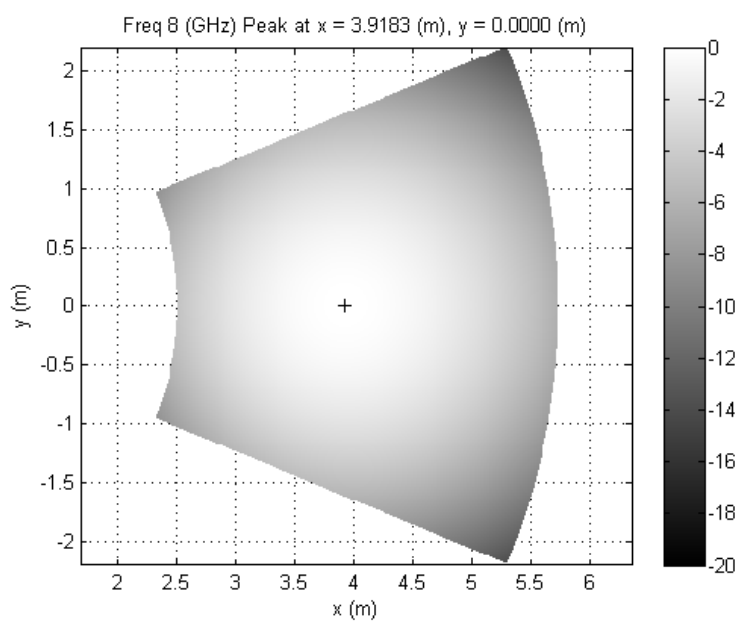

Fig. 2. Magnitude of illuminating fields over sector shaped reflector in $d B$.

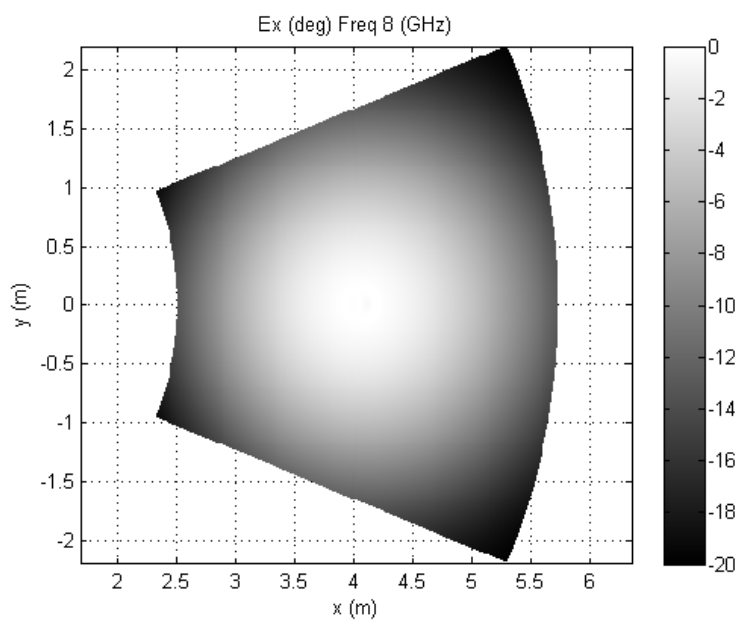

Fig. 3. Phase of x-polarised illuminating field over sector shaped reflector - note the supressed spherical phase factor.

In [5] we present the results of an extensive validation campaign for computing the $\mathrm{QZ}$ of a $17 \lambda$ serrated single offset CATR using five different asymptotic EM simulation methods for the CATR "Measurement" process:GO with GTD; Vector Huygens; Kirchhoff Huygens; Current Elements; FEKO (PO); were compared for accuracy with excellent agreement between all methods. In [17] the comparison is made between modelling a CATR (in this case a dual compensated system) using a full-wave method (FEKO employing the Multilevel Fast Multipole Method) and asymptotic method (PO/PTD). It is shown that for $1.5 \mathrm{GHz}$ ( $36 \lambda$ reflector CATR with $6-9 \lambda$ serrations) that the co-polar QZ cuts agree well but with more ripple detail shown with the full-wave results due to the improved modelling of the serrations and the ability for the full-wave model to account for all sources of illumination into the QZ as diffracted fields interact with the two reflectors. At $6 \mathrm{GHz}$ agreement between the methods is indistinguishable and here the full-wave method is at its limit with $4 \mathrm{~m}$ mesh points and $420 \mathrm{mins}$ computation time on a high-end work-station (the $1.5 \mathrm{GHz}$ results in comparison took $67 \mathrm{mins}$ ). The key advantage of using the full-wave solution was in the prediction of the very low cross-polar QZ field inherent in a compensated CATR (peak of $-40 \mathrm{~dB}$ ), which the asymptotic method could not achieve. For this work we need a simulation tool that permits the derivation of the error to signal level for a specified AUT and CATR combination. So for any reasonable gain antenna the AUT aperture averages our the fine detail of the CATR QZ ripple and hence the high performance offered by the full-wave approach is not required.

In this paper we thus choose to use the Current Element method which replaces the illuminating fields, shown above, with an equivalent surface current density $\underline{J_{S}}$ which is used as an equivalent source to the original fields. The surface current density across the reflector can then be obtained from the incident magnetic fields and the surface unit normal using the physical optics (PO) approximation [1, 5]. The elemental field within the CATR QZ can be obtained from the fields radiated by an electric current element. This is found from the vector potential and the free-space Green's function $[1,5]$. When the field point in the QZ is more than a few wavelengths from the radiating infinitesimal elemental source, the corresponding elemental electric fields can be obtained efficiently from the elemental magnetic fields using the far-field TEM condition with negligible approximation [5]. Thus, both the electric and magnetic fields can be obtained from the elemental surface current by integrating across the surface of the CATR reflector. In practice, for the case of a CATR with a QZ located at a distance from the, usually virtual, vertex $z$ that is larger than the focal length of the reflector, the error introduced by this plane-wave approximation is negligible. In this way the fields throughout the volume of space in front of the reflector (i.e. outside of the deep shadow region) can be computed efficiently and with enormous accuracy. To determine the quality of the CATR pseudo plane-wave it is customary for the amplitude and phases to be computed across a plane that is transverse to the range boresight. Figures 4 and 5 below present the amplitude of the principal and cross-polarised electric field for the horizontal and vertical cuts respectively through the centre of the $\mathrm{QZ}$ and at a frequency of $8 \mathrm{GHz}$. Here, the transverse plane is located at a distance of 1.136 times the focal length of the reflector from the virtual vertex, i.e. at $\mathrm{z}=6.13(\mathrm{~m})$. Here, the best fit $2^{\text {nd }}$ order polynomial which is used to determine the QZ taper is shown plotted using a dotted line across a larger $2 \mathrm{x}$ 
$2 \mathrm{~m} \mathrm{QZ} \mathrm{span} \mathrm{(note,} \mathrm{the} \mathrm{CATR} \mathrm{is} \mathrm{specified} \mathrm{for} \mathrm{a} 1 \mathrm{~m} \times 1 \mathrm{~m}$ QZ).

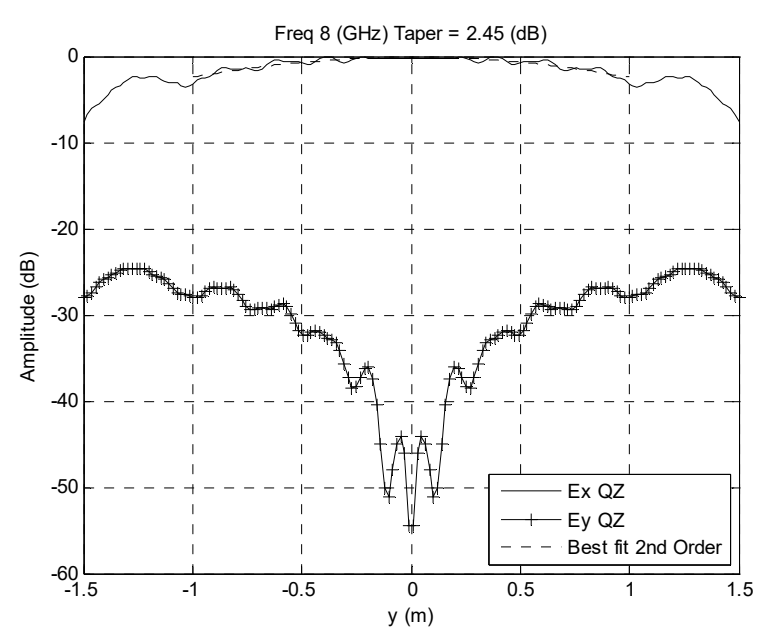

Fig. 4. Principal and cross-polar amplitude plotted across a vertical cut through the CATR QZ. black trace is $x$-pol field, black trace with + is y-pol field and dotted trace is least squares quadratic function denoting amplitude taper.

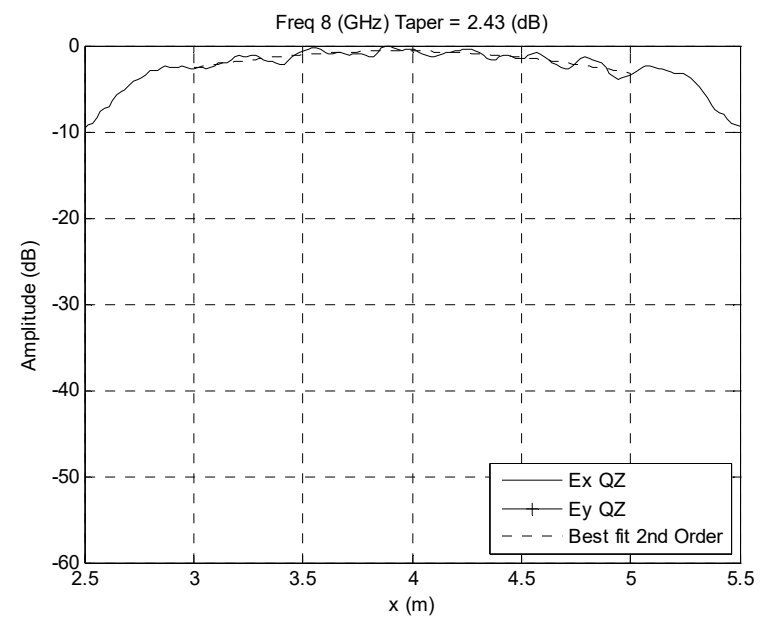

Fig. 5. Principal and cross-polar amplitude plotted across a horizontal cut through the CATR QZ, black trace is $x$-pol field, black trace with + is y-pol field and dotted trace is least squares quadratic function denoting amplitude taper.

Since the inception of the CATR, it has become customary to specify the performance in terms of amplitude taper, and amplitude \& phase ripple of this pseudo plane wave throughout a volume of space, termed the quiet zone (QZ). For the QMUL CATR the figures of merit for $1 \mathrm{~m} \mathrm{x} 1$ $\mathrm{m} \mathrm{QZ}$ at a distance $\mathrm{z}$ from Vertex to $\mathrm{QZ}$ of $6.13 \mathrm{~m}$ (i.e. $1.136 \times f)$ at $8 \mathrm{GHz}$ the $y$-axis amplitude taper was found to be $0.63 \mathrm{~dB}$, the $y$-axis peak to peak amplitude ripple was $1.04 \mathrm{~dB}$ and the $y$-axis peak to peak phase ripple was $5.86^{\circ}$. Conversely, the $x$-axis amplitude taper was $0.63 \mathrm{~dB}$ with the $x$-axis peak to peak amplitude ripple being $1.82 \mathrm{~dB}$ and the $x$-axis peak to peak phase ripple corresponding to $10.02^{\circ}$. The slightly degraded $x$-axis performance stems from the fact that the offset of the reflector lies within this plane. These values are very close to the aforementioned, industry accepted, specifications of $1 \mathrm{~dB}$ taper and $1 \mathrm{~dB}$ peak-to-peak amplitude ripple and $10^{\circ}$ peak-to-peak phase ripple. That being said and unfortunately in most cases, it is not directly apparent how a given QZ performance specification will manifest itself on the resulting far-field antenna pattern measurement. For this reason a new, sophisticated EM modelling tool was recently developed that allow "measured" far-field patterns to be produced for a given CATR AUT combination, with the computed coupling being based upon the reaction theorem. This is a well-known, general purpose, method for analysing coupling problems [15].

This theorem states that, provided the electric and magnetic field vectors $\left(\underline{E}_{1}, \underline{H}_{1}\right)$ and $\left(\underline{E}_{2}, \underline{H}_{2}\right)$ are of the same frequency and monochromatic then the mutual impedance, $Z_{21}$, between two radiators, antenna 1 (i.e. the CATR) and antenna 2 (i.e. the AUT), in the homogeneous environment described by $\varepsilon, \mu$ can be stated in terms of a closed surface integration. The mutual impedance will naturally be a function of the displacement between the respective antennas, their relative orientations, and their individual polarization properties. Once the mutual impedance, and therefore the mutual admittance is obtained, it is a simple matter to calculate the transmission scattering parameter $S_{21}$ that related the two coupled two-port scattering matrices. The elements $S_{12}=S_{21}$ are the complex transmission coefficients for the coupled antenna system which can be taken to represent a single point within the far-field "measurement". This integration can, in principle, be performed across the surface of any convenient free-space closed surface providing it encloses one or other of the antennas, however a great deal of computational ease can be sought if a spherical integrating surface is chosen to enclose the AUT.

Although in principal, any closed surface could be used, the advantage of adopting a spherical integration surface is that a general compound rotation can be implemented without the need to compute fields outside of this sampling interval. Such isometric vector rotations can be implemented using interpolation schemes (either rigorously or approximately [1]) or by expanding the fields onto a set of spherical vector mode functions and by rotating those functions [8]. When utilizing this technique to simulate CATR measurements it is crucial to recognise that the fields illuminating the AUT from the CATR only need to be computed once per frequency. This is very important as this can be a computationally intensive task (which for more complex CATR geometries could be produced using full-wave techniques as described earlier). This is also the case for the fields radiated by the AUT. Thus, the processing to calculate the "measured" antenna pattern is a very efficient task. In [5] we report a validation of this simulation tool that permits the derivation of the error to signal level for a specified AUT and CATR combination. It is demonstrated in [5] that equivalent multipath level (EMPL) between the actual AUT pattern and the simulated "measured" CATR pattern offers EMPL levels of order $50 \mathrm{~dB}$. Thus in addition to being able to provide conventional CATR QZ performance predictors such as amplitude taper, amplitude and phase peak-to-peak ripple, it is also possible to provide full-sphere simulated measured data for a given CATR AUT combination. We thus have an accurate tool that we can use to simulate the MARS technique in a CATR.

For a conventional CATR measurement, it is customary for the AUT to be carefully installed within the facility such that the AUT is situated about the axis of 
rotation. This has the effect of minimising the volume of the QZ needed as well as minimising the difference between the direct and indirect (i.e. scattered) illumination. However, and entirely contrary to usual antenna measurement practice, when taking a MARS type measurement $[9,10,11,12]$ we deliberately displace the AUT from the centre of rotation. This has the effect of maximising the differences in path length between the illuminating field and any scattered field. This makes the differences become far more pronounced than would otherwise be the case. And, it is exactly this more significant differentiation that enables their identification and subsequent extraction viable. For the case of a CATR measurement, unfortunately, this has the effect of increasing the size of the QZ that is needed to enclose a given AUT than would otherwise be the case. Thus, for the simulations performed here, the X-band SG90 pyramidal horn that was used as the test antenna was simulated located with $\mathrm{z}$-axis offset from the origin of the measurement coordinate system by $0.30 \mathrm{~m}, 0.61 \mathrm{~m}$ and $0.76 \mathrm{~m}$ for the three scenarios. In each case, the electromagnetic six-vector field was computed over the surface of a conceptual sphere with a radius of $0.91 \mathrm{~m}$ with a $1^{\circ}$ sample spacing in the $\theta$ and $\phi$ axis. The CATR pseudo TEM wave was then computed over a sphere of the same $0.91 \mathrm{~m}$ radius that was centred at the point $x=4.0 \mathrm{~m}, y=0.0 \mathrm{~m}, z=6.13 \mathrm{~m}$. The results of these simulated measurements and the FF-MARS processing can be seen presented in the following section. Note however, that the radius of the integrating sphere does not need to fit within the QZ of the CATR, instead it is the majority of the current sources that need to remain within the QZ of the CATR for the measurement to be valid. The size and shape of closed, but unbounded, integrating surface is unimportant providing the fields are sampled sufficiently finely across its surface so that the numerical evaluation of the reaction integral is reliable. This implies that, as we are evaluating a two-dimensional surface integral, selecting a larger integrating surface will result in longer computation times as we need to satisfy the sampling theorem across this surface requiring the evaluation of a greater number of data points.

\section{CATR Simulated FF-MARS measurements}

It has been long established that far-field MARS processing depends upon a measurement that is made with the AUT offset from the origin of the measurement coordinate system combined with a novel mode filtering algorithm [3, 4, 13]. In essence, once the far-field great circle antenna pattern cut has been acquired and the AUT mathematically translated back to the origin of the measurement coordinate system by means of a differential phase change $[1,3,4,13]$, the equivalent cylindrical mode coefficients (CMCs) can be deduced from far electric fields numerically using standard cylindrical near-field theory $[14$, $15,16]$. This procedure incorporates a highly efficient algorithm that relies upon an inverse fast Fourier transform (FFT). These CMCs can then be filtered using a carefully chosen band-pass filter function to remove artefacts that are not associated with the AUT whereupon the processed farfield pattern can be recovered from the reduced set of CMCs using a FFT based summation procedure. Details of the processing can be found in the open literature [1].

\subsection{Reflection Suppression}

Figure 6 below presents the CMCs as obtained from a simulated CATR measurement of a WR90 pyramidal horn that is shown in Figure 7. Here, the aperture of the horn was located at the origin of the measurement coordinate system. The dotted trace shows the equivalent CMCs that were computed from the simulated far-field "measurement". The highest order CMC that is associated with the AUT can be computed from the standard cylindrical sampling theorem as frequency and radius of conceptual cylinder that is centred about the rotation axis and is large enough to enclose the majority of the current sources $[1,8]$. The far-field data is sampled sufficiently finely to enable a large number of higher order modes to be computed however the finite size of the radiator means that only a subset of these modes is required to accurately represent the radiator explaining the limited span of the black trace, which represents this retained, finite, set of cylindrical modes.

The dotted-trace shown in Figure 7 presents the farfield pattern of this ideal measurement and represents the reference pattern, i.e. our "truth" model. A large amplitude scatterer was then introduced into the measurement simulation. This comprised a plane wave propagating at $30^{\circ}$ in azimuth with respect to the boresight direction of the range with maximum amplitude that was only $6 \mathrm{~dB}$ below than that of the CATR pseudo plane wave. The perturbed simulated measurement in the presence of this spurious signal can be seen plotted in Figure 7 as the black-trace. Finally, the black trace with dots represents the FF-MARS processed pattern. Here, the perturbed and FF-MARS processed traces are effectively identical. The absence in AUT offset in this simulated measurement means that the CMCs associated with the AUT and the scatterer are not displaced from one another when transformed to the cylindrical mode domain, as is apparent from inspection of Figure 6. Consequently, the band-pass filter that is applied in the mode domain does not attenuate the unwanted scattered fields.

However, Figure 9 contains an equivalent simulated measurement only in this case the AUT aperture has been displaced from the origin by $30 \mathrm{~cm}$ in z-axis. When translated back to the origin and transformed to the cylindrical mode domain, as is shown in Figure 8, it is clear that there is some degree of separation between the modes associated with the AUT (modes grouped around $n=0$ ) and those associated with the scatterer (the emergent group of modes centred around $n=30$ ). As before, from standard cylindrical near-field theory [1], it is well known that the highest order CMC that can be produced by a radiator when situated at the origin of the measurement coordinate system is $N=k_{0} \mathrm{a}=18$ in this case where $k_{0}$ is the free space propagation constant and $a$ denotes the radius of the minimum cylinder - as was defined above. Thus any higher order mode can be filtered out without affecting the properties of the underlying AUT irrespective of the complexity of that AUT pattern. Consequently, when filtered and transformed back to the angular domain, there is correspondingly some degree of suppression of the scattered signal. Here in Figure 9, it is clear that the FF-MARS processed pattern (solid line with doted markers) is in better agreement with the reference pattern (dotted-trace).

However, as the mode spectra are not completely separated, $c f$. Figure 8, the MARS suppression is not 
complete. Thus, as the AUT is successively translated away from the origin of the measurement coordinate system, as the results in Figures 11 and 13 illustrate, the degree of mode separation, (i.e. orthogonalisation) increases, as is confirmed by inspection of Figures 10 and 12. Thus, the spurious scattered signal is attenuated ever more effectively as the displacement is increased until the respective mode spectra do not overlap and are effectively orthogonalised. From inspection of Figure 13 it is quite clear that the FFMARS processing is having a very significant effect on the far-field pattern and is very effectively suppressing the artefacts that are associated with the spurious scatterer as the solid trace with dotted markers (FF-MARS processed pattern) is in very close agreement with dotted (reference) trace. Some small differences are evident in regions of very low field intensity, i.e. for parts of the pattern that are below, for example, $-50 \mathrm{~dB}$.

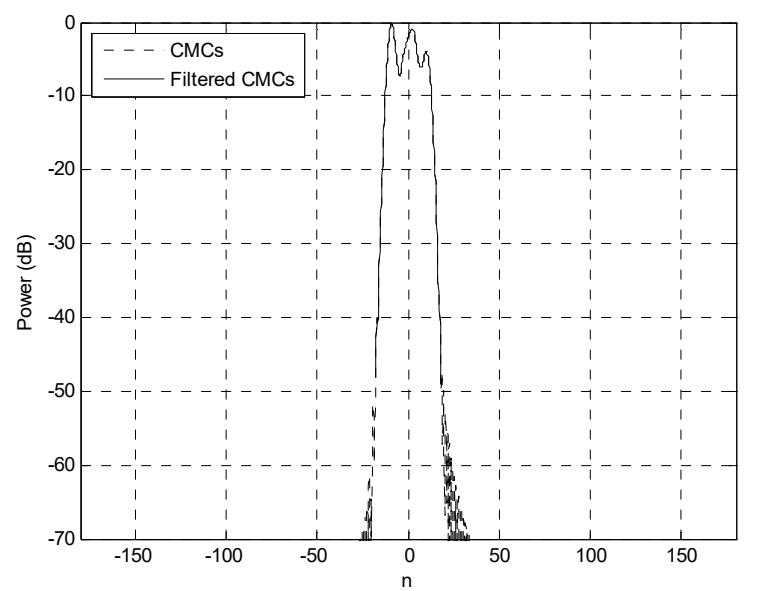

Fig. 6. Cylindrical mode coefficients for $0 \mathrm{~m}$ AUT offset case

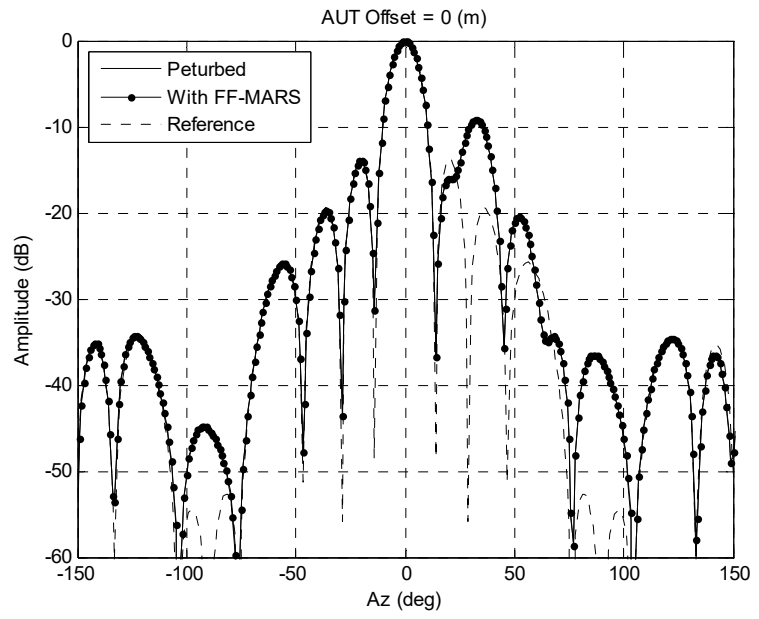

Fig. 7. Far-field amplitude pattern for $0 \mathrm{~m}$ AUT offset case

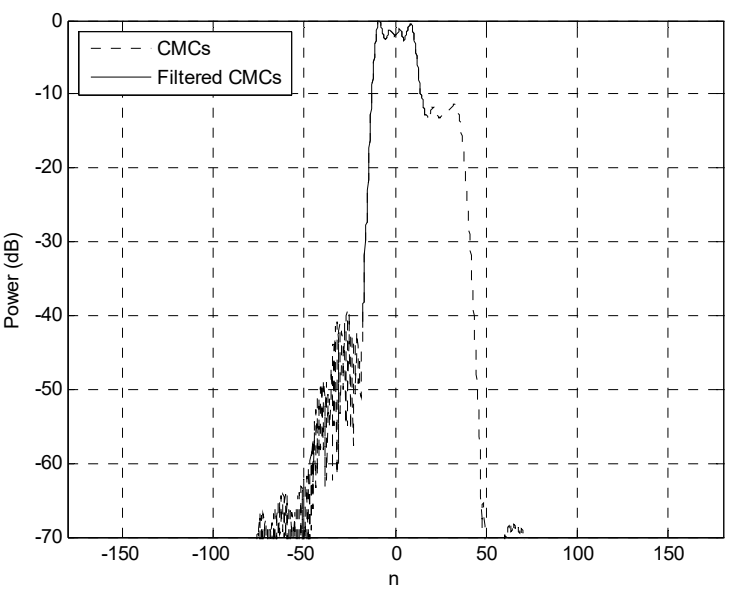

Fig. 8. Cylindrical mode coefficients for $0.30 \mathrm{~m}$ AUT offset case.

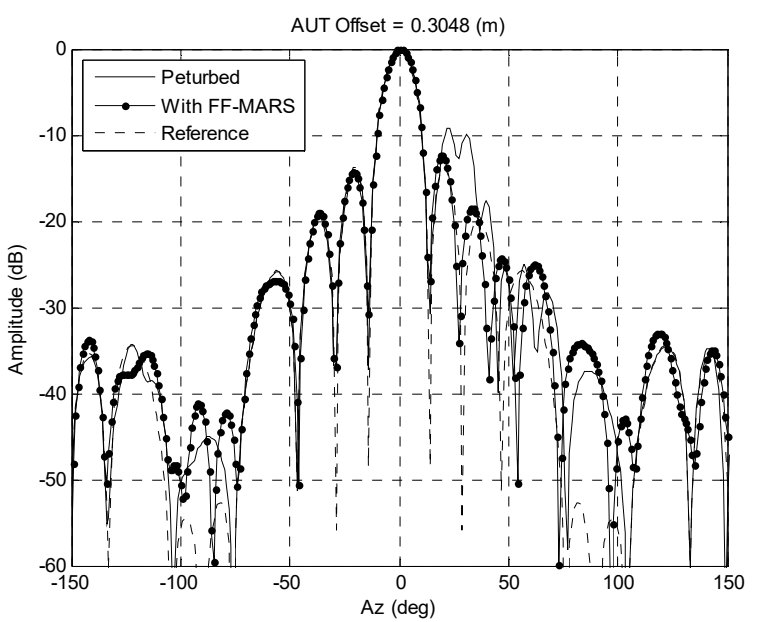

Fig. 9. Far-field amplitude pattern for $0.30 \mathrm{~m}$ AUT offset case

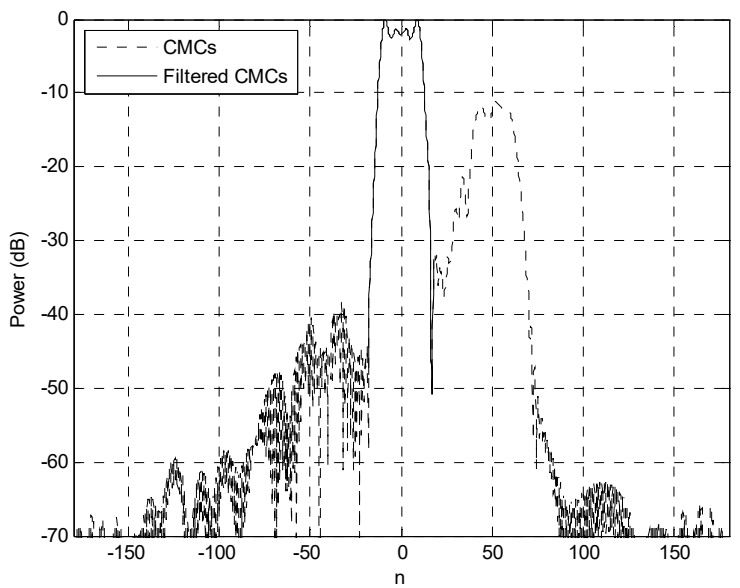

Fig. 10. Cylindrical mode coefficients for $0.61 \mathrm{~m}$ AUT offset case. 


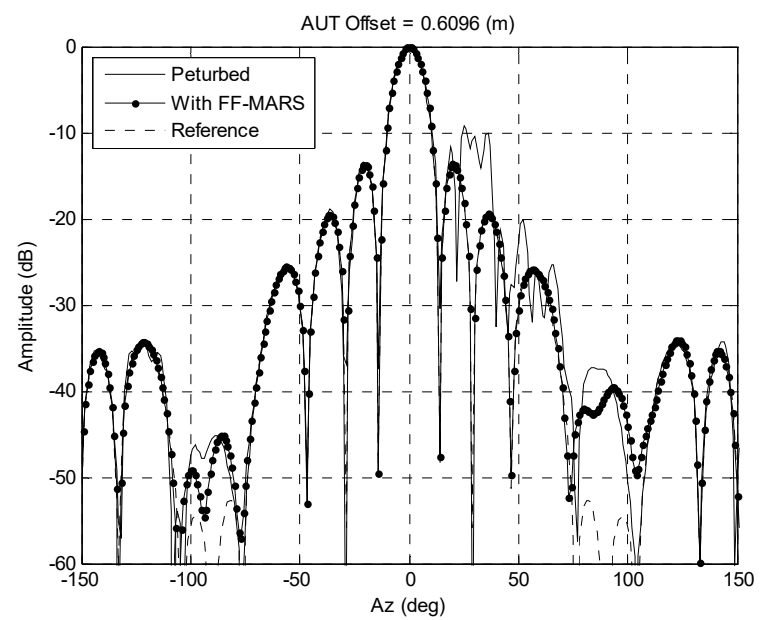

Fig. 11. Far-field amplitude pattern for $0.61 \mathrm{~m}$ AUT offset case.

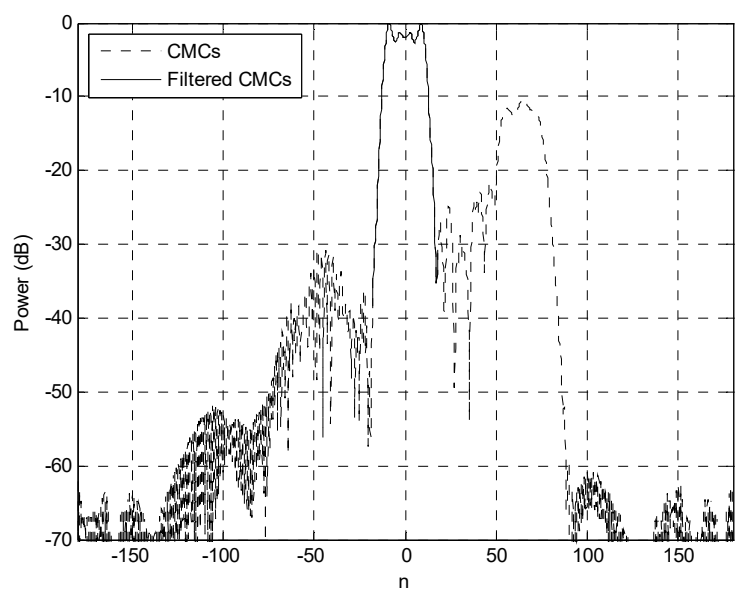

Fig. 12. Cylindrical mode coefficients for $0.76 \mathrm{~m}$ AUT offset case.

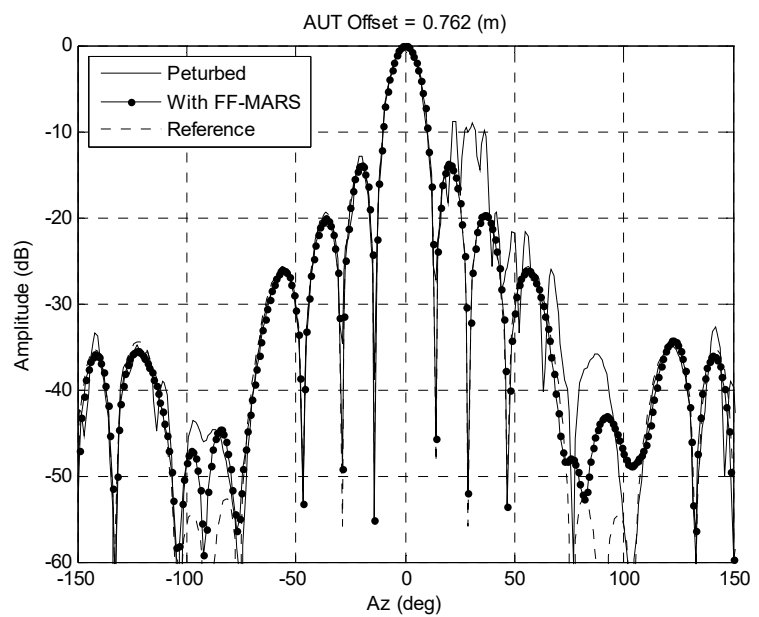

Fig. 13. Far-field amplitude pattern for $0.76 \mathrm{~m}$ AUT offset case.

It is also interesting to note how translating the AUT across progressively larger regions of the CATR QZ also increase the general error level of measurement. The predicted CATR pseudo TEM wave used within these measurement simulations includes amplitude taper stemming from the feed pattern and amplitude and phase ripple primarily emanating from fields diffracting from the knife-edge of the CATR reflector both of which mean that the QZ is of a finite quality and size, $c f$. Figures 4 and 5 above. Thus, as the AUT is progressively offset from the origin the measurement also occupies a larger and larger region of space placing ever greater demands upon the quality of the CATR QZ. This means that although the effectiveness of the FF-MARS processing increases as the AUT offset is increased, other CATR QZ related errors increase eventually compromising the measurement technique. Although not treated within these simulations, positioner alignment is also known to become more critical as the magnitude of the AUT offset increase [1]. Thus, although it is preferable to offset the AUT by an amount that is equivalent to the maximum dimension of the AUT, as this is an engineering compromise between maximising suppression and minimising CATR QZ with larger translations is generally being undesirable.

As an additional test, the $0.61 \mathrm{~m}$ AUT offset case was reprocessed using a cosine squared window function. This mode filter imposes less of a discontinuity in the mode domain than the brick-wall band pass filter that is often used and has been seen to provide a minor improvement in the MARS filtered far-field patterns [7].

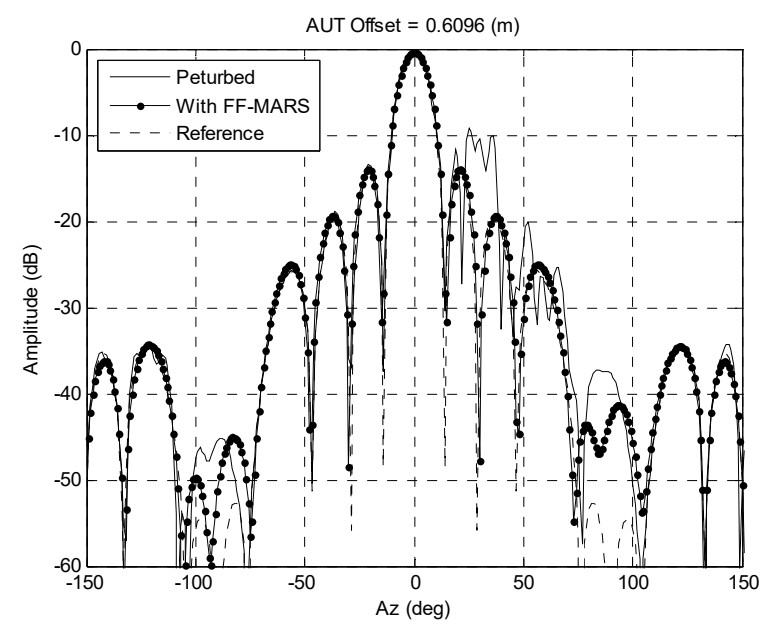

Fig. 14. Far-field amplitude pattern for $0.61 \mathrm{~m}$ AUT offset case with cosine squared CMC window function.

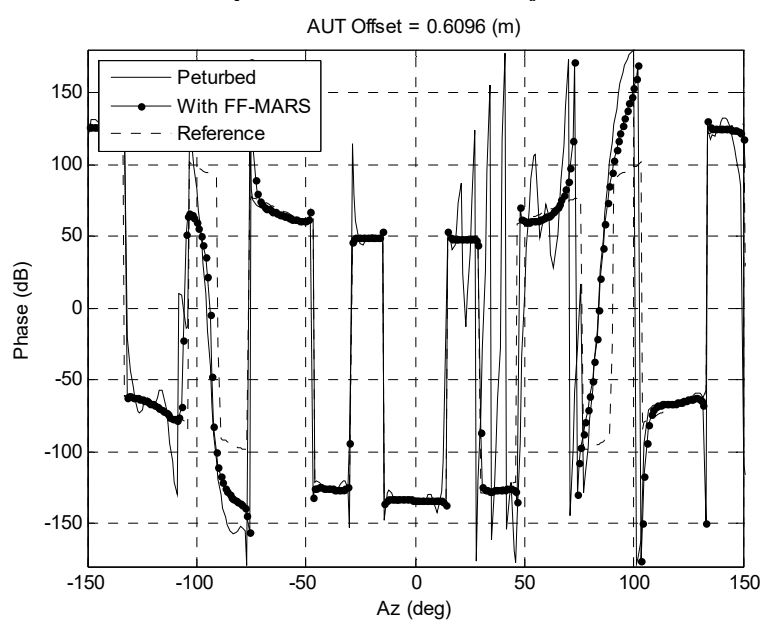

Fig. 15. Far-field phase pattern for $0.61 \mathrm{~m}$ AUT offset case with cosine squared $C M C$ window function.

From comparison of Figure 14 and Figure 11 it can be seen that the degree of agreement attained between the reference pattern and the MARS filtered pattern is 
marginally improved, especially for the back-lobes. For the sake of completion Figure 14 presents a comparison of the reference and MARS filtered phase plots. Here, it can be seen that the agreement attained is very encouraging everywhere except the region of low amplitude where it is more difficult to control phase, i.e. in the null region around $\pm 90^{\circ}$.

\subsection{Feed spill-over suppression}

After range reflections, often the next most significant contributor to the quality of the CATR QZ is the interference of direct illumination of the QZ by the feed. Direct illumination of the CATR quiet-zone is usually minimized through the use of absorber collars on the feed and/or baffles positioned within the chamber [1]. However feed spill-over effects are seen, to some extent, in many ranges with the widely employed offset reflector configuration and low gain feed making this to some extent almost unavoidable with dual reflector CATRs being perhaps the most susceptible designs.

Typically CATR QZ field probing involves using a planar near-field scanning system [1] to measure the horizontal and vertically polarized components of the electric field across a plane that is transverse to the boresight, i.e. $\mathrm{z}$-axis, of the range. It can be seen that the $x$ - and $y$ polarized components of the field radiated by the feed in the CATR QZ are generally comparatively small (e.g. $40 \mathrm{~dB}$ down) when compared to the pseudo plane-wave formed by reflection from the reflector itself. However the $z$-polarized component of the field radiated by the feed in CATR QZ, which in practice is not normally characterized during range commissioning or validation activities, can be significant and in this case was circa $13 \mathrm{~dB}$ greater than the longitudinal component of the pseudo plane wave thereby dominating this polarization. Figure. 16 shows the amplitude of the $\mathrm{z}-$ polarized electric field radiated by the feed in the CATR QZ where the levels have been plotted normalized relative to the peak of the pseudo plane wave, i.e. the $E_{x}$ component in this case.

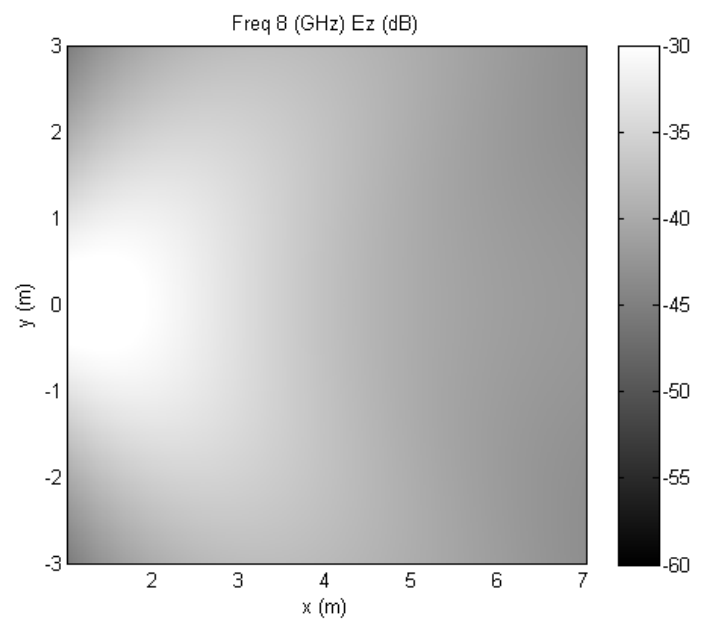

Fig. 16. Z-component of field radiated by feed in CATR $Q Z$ plotted across a plane transverse to the range boresight.

Crucially, it is the entire electromagnetic six-vector of the pseudo plane wave formed by the CATR that couples into the aperture of the test antenna that produces the measured far-field antenna pattern $[1,5]$ function. The actual coupling can be determined using the reaction integral which is based upon a reciprocity relationship. This suggests that on boresight feed spill-over related effects could be comparatively small. However, as the AUT is rotated so that its aperture aligns more closely with the zaxis of the range then the coupled power can increase meaning that feed spill-over effects become far more pronounced on the wide out antenna pattern function.

The existing QMUL CATR EM model, as presented above, was used to compute the fields radiated by the CATR at $8 \mathrm{GHz}$ with and without direct illumination of the QZ by the feed. The coupling of these fields into the AUT was then obtained from a numerical evaluation of the reaction integral which produced a simulated CATR measurement. As before the AUT was offset from the origin of the measurement coordinate system by an amount that was larger than the maximum dimension of the AUT and in this case a $0.61 \mathrm{~m}$ displacement in the $\mathrm{z}$-axis was used which is at a normal to the aperture plane of the AUT which is also orthogonal to the vertical azimuth rotation axis of the CATR. Respectively, Figure 17 and Figure 18 present azimuth and elevation far-field AUT patterns.

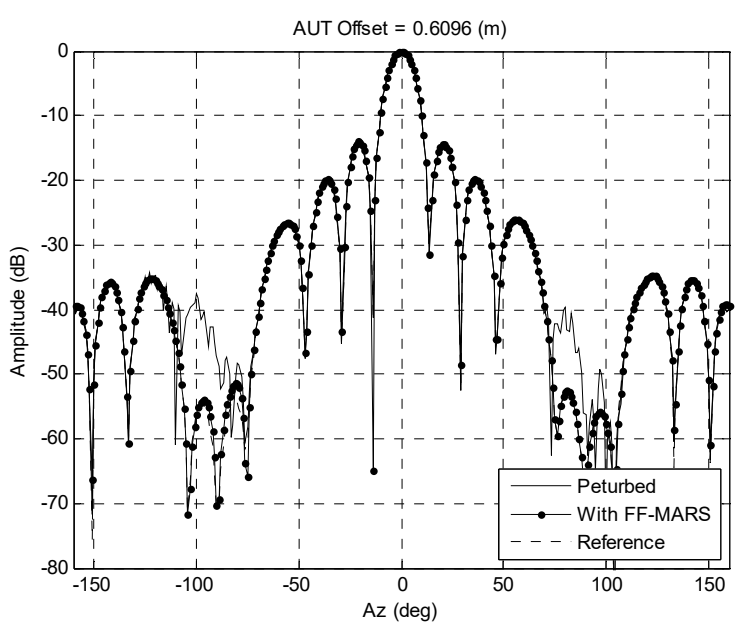

Fig. 17. Far-field azimuth cut showing feed spill-over and it suppression with MARS processing.

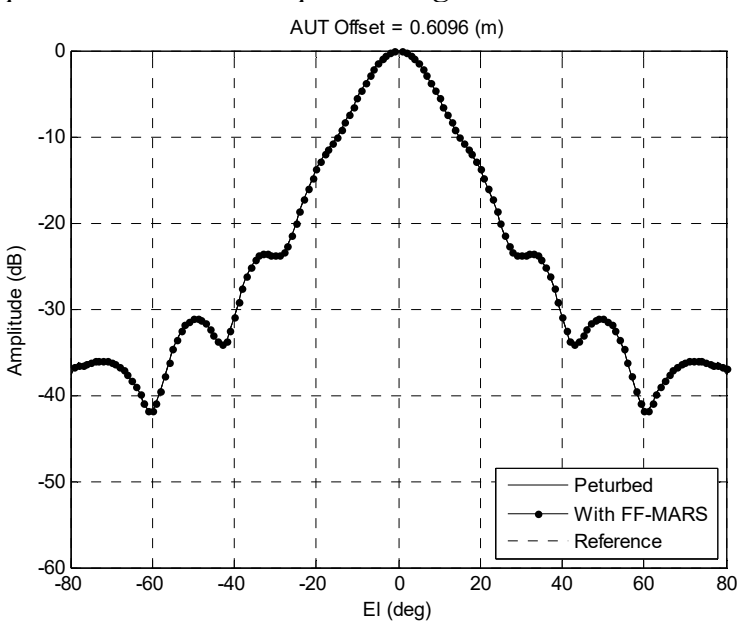

Fig. 18. Far-field elevation cut showing very little effect arising from feed spill-over.

Here, the dotted trace denotes the reference ideal farfield patterns which are shown plotted against the perturbed simulated "measured" fields which are denoted by the black traces. As expected, these differ most significantly in the 
region of the wide-out antenna side-lobes. Standard FFMARS processing was again used to suppress the effects of the feed spill-over with the results of the FF-MARS processing being denoted with the solid trace with dotted markers. As expected feed spill-over had the largest impact in the horizontal plane as this is the plane in which the feed was offset. From inspection of Figure 17, it is clear that the FF-MARS processing has significantly reduced the effect of the feed spill-over (by circa $15 \mathrm{~dB}$ around $\pm 90^{\circ}$ ) with the reference and FF-MARS processed patterns yielding very good agreement.

\section{Summary and Conclusion}

A sophisticated EM model of the CATR measurement process has been used to investigate the impact of various measurement error terms within the error budget of an offset reflector CATR. This model has been used to recreate a number of conventional FF-MARS measurements whereupon similar phenomena have been observed in the EM model as have been previously noted with actual CATR measurements. Specifically these are: 1) the effects of scattering on a far-field pattern depend upon the AUT displacement, with larger displacements resulting in higher angular frequency ripple on the far-field patterns, 2) CMCs resulting from scattering are displaced to higher order modes, with AUT modes being displaced to lower order modes when the AUT is mathematically translated to the origin of the measurement co-ordinate system, 3) the amount of separation between mode spectra associated with scattering and those associated with the AUT increases as the displacement increases, and 4) FF-MARS is capable of effectively suppressing scattering providing the magnitude of the displacement is sufficiently large. This behaviour has also been observed when using spherical mode based expansions, $c f$. $[1,9,10], 5)$ FF-MARS is capable of suppressing CATR feed spill-over, which can be a major issue particularly in dual reflector CATRs.

As shown above, the CATR EM model has been able to provide further confirmation of the effectiveness of the FF-MARS processing showing that it can be used with a high degree of confidence. All the steps used within the acquisition and post-processing are in common with the well-known and well understood principles of measurement theory, and all results obtained to date have attested to the success of the method. The AUT displacement and the correspondingly finer sample point spacing are acceptable providing the sampling criterion is adhered to. The mathematical displacement of origins of the far-field data to the measurement origin is implemented rigorously, the selection of the cylindrical mode filter being defined by the frequency, physical size of the antenna and its conceptually idealised measurement location. The final result with FFMARS processing can be degraded if the translation of the AUT is incorrectly applied, or the mode filter is too tight, however, importantly, these parameters are controlled by the user. Furthermore, results of a FF-MARS measurement have been presented showing for the first time the quantitative impact of feed spill-over on a CATR measurement. We have been able to show that this effect is primarily due to the $z$-component of the quiet zone field, $c f$. Figure 16, generated by the feed and that this component contributes to the measured field when the AUT points significantly away from boresight. Additionally, it was demonstrated that FF-MARS processing can be used to suppress these effects with results that very nearly remove the effect completely.

Finally, as this paper summarises the findings of an on-going programme of research, the future plans include investigating the effectiveness of FF-MARS in supressing other non-physical measurement artefacts that stem from imperfections within the CATR pseudo-plane wave. This paper should encourage antenna measurement practitioners that MARS can be effectively used in a CATR and it provides quantitative evidence of its validity, complementing other published work of a more qualitative form reporting its performance using purely measured data.

\section{References}

[1] Parini, C.G., Gregson, S.F., McCormick, J., Janse van Rensburg, D.: "Theory and Practice of Modern Antenna Range Measurements", IET Press, 2014, ISBN 978-1-84919-560-7.

[2] Newell, A.C.: "Error Analysis Techniques for Planar Near-field Measurements", IEEE Transactions on Antennas and Propagation, vol. AP-36, pp. 754-768, June 1988.

[3] Gregson, S.F., Williams, B., Masters, G.F., Newell, A.C., Hindman, G.E.: "Application of Mathematical Absorber Reflection Suppression To Direct Far-Field Antenna Range Measurements", AMTA, October, 2011.

[4] Gregson, S.F., Dupuy, J., Parini, C.G., Newell, A.C., Hindman, G.E.: "Application of Mathematical Absorber Reflection Suppression to Far-Field Antenna Measurements", LAPC November, 2011.

[5] Parini, C.G., Dubrovka, R., Gregson, S.F.: “CATR Quiet Zone Modelling and the Prediction of "Measured" Radiation Pattern Errors: Comparison using a Variety of Electromagnetic Simulation Methods" AMTA October 2015.

[6] Olver, A.D., Parini, C.G.: "Millimeterwave Compact Antenna Test Ranges", JINA November 1992, Nice.

[7] Gregson, S.F., Newell, A.C. , Hindman, G.E.: "Examination of Far-Field Mathematical Absorber Reflection Suppression Through Computational Electromagnetic Simulation", International Journal of Antennas and Propagation, Special Issue on Recent Advances in Near-Field to Far-Field Transformation Techniques, March 2012.

[8] Hansen, J.E. (Ed.): "Spherical Near-Field Antenna Measurements", IEE Electromagnetic Waves Series 26, ISBN 086341 110X, 1988.

[9] Hindman, G.E., Newell, A.C.: "Spherical NearField Self-Comparison Measurements", AMTA 26th Annual Meeting \& Symposium, Atlanta, GA, October 2004.

[10]Hindman, G.E., Newell, A.C.: "Reflection Suppression in a large spherical near-field range", AMTA 27th Annual Meeting \& Symposium, Newport, RI, October. 2005.

[11]Hindman, G.E., Newell, A.C.: "Reflection Suppression To Improve Anechoic Chamber Performance", AMTA Europe 2006, Munich, Germany, March 2006.

[12] Hess, D.W.: "The IsoFilterTM Technique: Isolating an Individual Radiator from Spherical Near-Field Data Measured in a Contaminated Environment", EuCAP 2007.

[13] Gregson, S.F., Newell, A.C., Hindman, G.E.: "Behaviour of Orthogonal Wave Functions And The Correction of Antenna Measurements Taken in 
Non-Anechoic Environments", LAPC, 11-12

November 2013, Loughborough, UK.

[14] Gregson, S.F., Newell, A.C., Hindman, G.E.:

"Reflection Suppression in Cylindrical Near-Field Antenna Measurement Systems - Cylindrical MARS", AMTA 31st Annual Meeting \& Symposium, Salt Lake City, UT, November 2009.

[15] Gregson, S.F., Newell, A.C., Hindman, G.E.: "Reflection Suppression in Cylindrical Near-Field Measurements of Electrically Small Antennas", Loughborough Antennas \& Propagation Conference, November, 2009.

[16] Gregson, S.F., Newell, A.C., Hindman, G.E. Carey, M.J.: "Advances in Cylindrical Mathematical Absorber Reflection Suppression", 4th European Conference on Antennas and Propagation, Barcelona, 12th -16th April, 2010.

[17] Rodriguez, J., Geise, A., Schmidt, C. H., Migl, J., Steiner, H.-J.: "Asymptotic and full wave simulation models for compensated compact range design and analysis," in Antennas and Propagation Conference (LAPC), 2013 Loughborough, Nov. 2013, pp. 461-466. 